\title{
Sacral insufficiency fractures after lumbosacral arthrodesis: salvage lumbopelvic fixation and a proposed management algorithm
}

\author{
Thomas J. Buell, MD, ${ }^{1}$ Ulas Yener, MD, ${ }^{1}$ Tony R. Wang, MD, ${ }^{1}$ Avery L. Buchholz, MD, MPH, ${ }^{1}$ \\ Chun-Po Yen, MD, ${ }^{1}$ Mark E. Shaffrey, MD, ${ }^{1}$ Christopher I. Shaffrey, MD, ${ }^{2}$ and \\ Justin S. Smith, MD, PhD' \\ 1Department of Neurological Surgery, University of Virginia Health System, Charlottesville, Virginia; and 2Department of \\ Neurological Surgery, Duke University Medical Center, Durham, North Carolina
}

\begin{abstract}
OBJECTIVE Sacral insufficiency fracture after lumbosacral (LS) arthrodesis is an uncommon complication. The objective of this study was to report the authors' operative experience managing this complication, review pertinent literature, and propose a treatment algorithm.

METHODS The authors analyzed consecutive adult patients treated at their institution from 2009 to 2018. Patients who underwent surgery for sacral insufficiency fractures after posterior instrumented LS arthrodesis were included. PubMed was queried to identify relevant articles detailing management of this complication.
\end{abstract}

RESULTS Nine patients with a minimum 6-month follow-up were included (mean age $73 \pm 6$ years, BMl $30 \pm 6 \mathrm{~kg} / \mathrm{m}^{2}$, $56 \%$ women, mean follow-up 35 months, range 8-96 months). Six patients had osteopenia/osteoporosis (mean dual energy x-ray absorptiometry hip T-score $-1.6 \pm 0.5)$ and 3 received treatment. Index LS arthrodesis was performed for spinal stenosis $(n=6)$, proximal junctional kyphosis $(n=2)$, degenerative scoliosis $(n=1)$, and high-grade spondylolisthesis $(n=1)$. Presenting symptoms of back/leg pain $(n=9)$ or lower extremity weakness $(n=3)$ most commonly occurred within 4 weeks of index LS arthrodesis, which prompted CT for fracture diagnosis at a mean of 6 weeks postoperatively. All sacral fractures were adjacent or involved S1 screws and traversed the spinal canal (Denis zone III). H-, U-, or T-type sacral fracture morphology was identified in 7 patients. Most fractures $(n=8)$ were Roy-Camille type II (anterior displacement with kyphosis). All patients underwent lumbopelvic fixation via a posterior-only approach; mean operative duration and blood loss were 3.3 hours and $850 \mathrm{ml}$, respectively. Bilateral dual iliac screws were utilized in 8 patients. Back/leg pain and weakness improved postoperatively. Mean sacral fracture anterolisthesis and kyphotic angulation improved (from $8 \mathrm{~mm} / 11^{\circ}$ to $4 \mathrm{~mm} / 5^{\circ}$, respectively) and all fractures were healed on radiographic follow-up (mean duration 29 months, range 8-90 months). Two patients underwent revision for rod fractures at 1 and 2 years postoperatively. A literature review found 17 studies describing 87 cases; potential risk factors were osteoporosis, longer fusions, high pelvic incidence (PI), and postoperative PI-to-lumbar lordosis (LL) mismatch.

CONCLUSIONS A high index of suspicion is needed to diagnose sacral insufficiency fracture after LS arthrodesis. A trial of conservative management is reasonable for select patients; potential surgical indications include refractory pain, neurological deficit, fracture nonunion with anterolisthesis or kyphotic angulation, L5-S1 pseudarthrosis, and spinopelvic malalignment. Lumbopelvic fixation with iliac screws may be effective salvage treatment to allow fracture healing and symptom improvement. High-risk patients may benefit from prophylactic lumbopelvic fixation at the time of index LS arthrodesis.

https://thejns.org/doi/abs/10.3171/2019.12.SPINE191148

KEYWORDS adult spinal deformity; complications; iliac screws; lumbosacral junction; pseudarthrosis; rod fracture; sacral fracture; scoliosis

\footnotetext{
ABBREVIATIONS DEXA = dual energy x-ray absorptiometry; $\mathrm{DF}=$ dorsiflexion; $\mathrm{LL}=$ lumbar lordosis; $\mathrm{LS}=$ lumbosacral; $\mathrm{PF}=$ plantarflexion; $\mathrm{PI}=$ pelvic incidence; $\mathrm{PJK}=$ proximal junctional kyphosis; rhBMP-2 = recombinant human bone morphogenetic protein-2; TLIF = transforaminal lumbar interbody fusion; UIV = upper-most instrumented vertebra.
}

SUBMITTED September 22, 2019. ACCEPTED December 31, 2019.

INCLUDE WHEN CITING Published online March 27, 2020; DOI: 10.3171/2019.12.SPINE191148. 
$\mathrm{T}$ RAUMATIC sacral fractures are a rare entity thought to be often overlooked and underdiagnosed, even when there are other obvious pelvic injuries. . $^{6,16,31,36}$ Stress or fragility fractures of the sacrum, which typically affect postmenopausal women with osteoporosis, may be even more challenging to diagnose due to lack of antecedent trauma. ${ }^{4,11,18,23,30}$ Sacral stress fractures have also been termed "insufficiency" fractures because the injury represents spontaneous failure of bone biomechanics under normal physiological loads. ${ }^{14,18}$ As such, positive risk factors associated with sacral insufficiency fractures include senile osteopenia, postmenopausal osteoporosis, steroidinduced osteoporosis, and prior radiation therapy. ${ }^{4,11,18,30}$ Fracture symptoms can be insidious and nonspecific; patients may present with diffuse low-back pain, leg pain, and sacral pain or tenderness..$^{18,30}$ Radiographs may poorly demonstrate the osteoporotic sacral fracture; therefore, a high index of suspicion is needed to prompt further investigation. CT imaging or radionuclide bone scans can help to diagnose this entity. ${ }^{4,11,30}$

Sacral insufficiency fractures below posterior segmental spinal instrumentation are an uncommon postoperative complication. ${ }^{2,8,9,12-15,20,22,25-28,33,37-39}$ Although the pathophysiology of this complication is incompletely understood, some biomechanical studies have indicated postoperative increases in stress transmission and alterations in load distribution involving the sacrum..$^{14,40}$ Klineberg et al. described potential sacral stress risers after posterior lumbosacral (LS) arthrodesis; these include the implant system (e.g., fixed-angle screw systems may excessively preload sacral screws) and surgical techniques (e.g., sacral alar decortication for graft incorporation may induce a starting point for vertical shear strain). ${ }^{14}$ In a separate study, Odate et al. suggested patients with high pelvic incidence (PI) and large postoperative PI-to-lumbar lordosis (LL) mismatch may have increased sacral force transmission from compensatory pelvic retroversion. $^{26}$

Spine patients affected by this complication are often older and may not tolerate prolonged immobilization and bedrest from intractable fracture pain. ${ }^{22,26}$ Therefore, timely diagnosis and appropriate management are critical, and may facilitate early recovery of independent ambulation and functional autonomy. ${ }^{22,26}$ The literature describing sacral fractures after LS arthrodesis is limited to mostly case reports and small, single-center cohort studies. ${ }^{2,8,9}$, 12-15,20,22,25-28,33,37-39 Also, there is lack of consensus regarding optimal management. Therefore, the objective of this study was to report our operative experience treating this complication, review pertinent literature, and propose a treatment algorithm.

\section{Methods}

\section{Patient Cohort and Data Collection}

Following IRB approval, we reviewed a prospectively collected database of consecutive patients who underwent spine surgery at the University of Virginia Health System Department of Neurological Surgery from 2009 to 2018. For this study, we included all patients with sacral fractures after LS arthrodesis who underwent operative treat- ment. Patients with sacral fractures caused by high-impact trauma or tumor were excluded. The minimum duration of follow-up for study inclusion was 6 months. Baseline patient data included demographics, comorbidities, lumbar spine surgery history, and details of the index LS arthrodesis operation. Metabolic bone disease (i.e., osteopenia/ osteoporosis) was assessed based on medical chart review, history of previous osteoporotic fractures, and dual energy $\mathrm{x}$-ray absorptiometry (DEXA) results according to $\mathrm{WHO}$ criteria. Any treatments that patients underwent for osteoporosis were recorded.

Diagnosis of sacral insufficiency fracture was determined based on CT. Data related to clinical presentation included patient symptoms, details of neurological deficits, and the postoperative time after index LS arthrodesis to symptom onset. Sacral fractures were classified according to Denis et al., Roy-Camille et al., and Strange-Vognsen and Lebech classifications (Fig. 1A and B), 6,31 Fracture shape morphology (e.g., "H" or "U") was assessed using coronal CT reconstructions. ${ }^{16}$ Sacral fracture anterior-posterior translation and kyphotic angulation were measured using sagittal CT reconstructions and methodology described by Kuklo et al. (Fig. 1C). ${ }^{16}$ Data regarding salvage operative treatment included revision construct proximal and distal segmental levels, pelvic fixation techniques, use of osteobiologics, pedicle screw replacement/upsizing, levels of decompression, operative blood loss, operative duration, and associated complications. Our operative technique is described below.

\section{Lumbopelvic Fixation as Salvage Treatment Modality}

Our general indications for surgical management of sacral insufficiency fractures after LS arthrodesis include refractory pain, neurological deficits, and fracture instability with severe displacement/angulation. We generally utilize a posterior-only approach for neural decompression, instrumentation revision, fracture reduction, and sacral stabilization with extension of the construct distally using bilateral iliac screws for pelvic fixation. We typically implement "quad-iliac bolt constructs" with bilateral

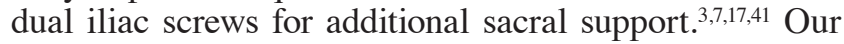
technique for iliac screw placement has been detailed in a prior publication. ${ }^{24}$ Of note, it is important to deeply recess all iliac screws so that the screw head is at least flush with the lateral aspect of the ilium to reduce risk of implant prominence. ${ }^{24}$ To implement quad-iliac screw constructs, we leave enough distal rod "overhang" below the sacral S1 screw head so that two iliac screw connectors can be placed in side-by-side fashion. Post-LS fusion sacral insufficiency fractures typically involve S1 screws and there may be varying degrees of screw loosening and/or pullout. Fracture reduction/stabilization involves removal and replacement, when possible, of involved pedicle screws. During this process, we attempt to improve the revision construct's biomechanical strength by upsizing pedicle screws. ${ }^{19,29}$ Revision sacral fixation is performed at the surgeon's discretion and depends on the integrity of this structure. Next, sacral decompression can be performed if there is evidence of neural compromise at the fractured level. Then morselized local bone (e.g., iliac crest bone graft), cancellous allograft bone, and recombinant human 


\section{A. Denis Classification}

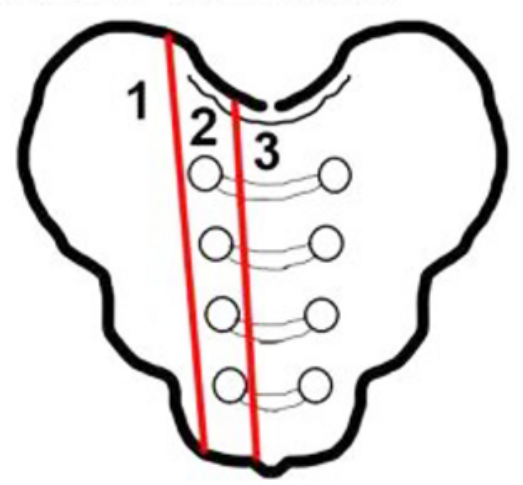

\section{B. Modified Roy-Camille and Strange-Vognsen and Lebech Classification}
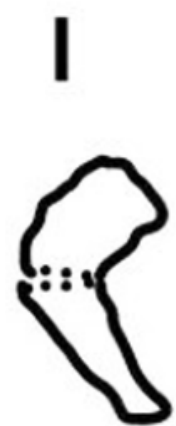
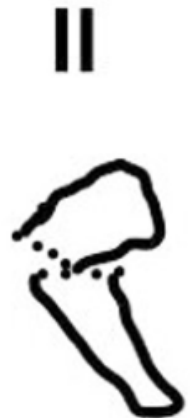

III

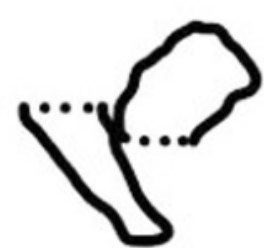

IV

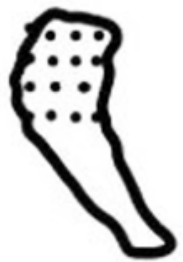

\section{Kuklo Sacral Fracture Displacement and Angulation}

\section{Lordosis (negative)}
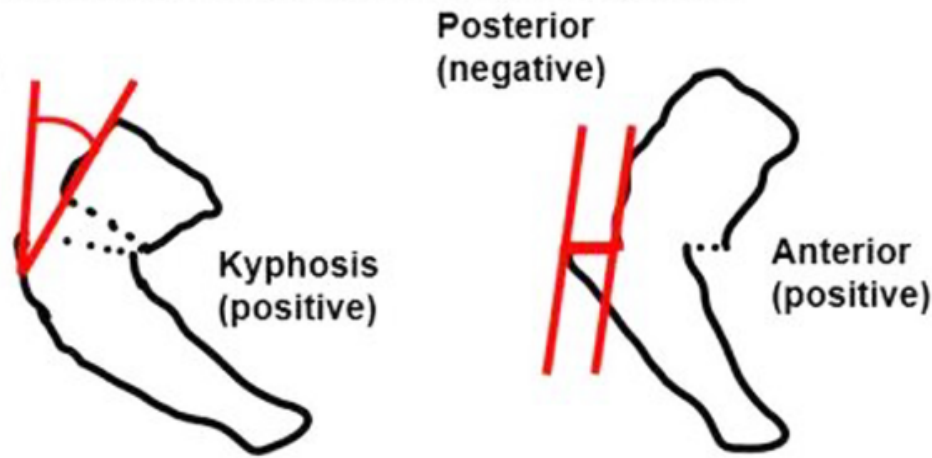

FIG. 1. A: Denis classification. In 1988, Denis et al. described three different sacral injury zones: zone I (the alar region lateral to the foramina), zone II (the foraminal region without central canal involvement), and zone III (the region of the central sacral canal). ${ }^{6}$ Denis zone III injuries are so-called "transverse sacral fractures" that primarily involve the central sacral canal, but the fracture line may traverse the other two zones. Of note, complex fractures with both longitudinal/vertical and horizontal/transverse components (e.g., fractures with $\mathrm{H}$ - or U-shape morphology) are, by definition, Denis zone III injuries. ${ }^{16} \mathrm{~B}$ : Modified Roy-Camille and StrangeVognsen and Lebech classification. Subclassification of transverse fractures of the upper sacrum (i.e., Denis zone III injuries) by Roy-Camille et al. ${ }^{31}$ and Strange-Vognsen and Lebech: ${ }^{36}$ type I (flexion injury with simple anterior bending without translation), type II (flexion injury with angulated, posteriorly displaced upper sacral fragment), type III (extension injury with complete anterior displacement of upper sacral fragment that slips downward in front of lower sacral fragment, more or less vertical orientation), and type IV (axial-loading injury with complete comminution of upper sacral fragment without displacement from the lower fragment). Of note, for this study we categorized angulated sacral fractures with either anterior or posterior translation as Roy-Camille type II, as long as translation did not result in a complete slip. C: Kuklo sacral fracture displacement and angulation. Transverse sacral fracture anterior-posterior translation and kyphotic angulation based on Kuklo et al. measurement methodology and sagittal CT reconstructions. ${ }^{16}$ Figure is available in color online only.

bone morphogenetic protein-2 (rhBMP-2) can be meticulously packed across fracture lines to promote arthrodesis and healing. Depending on the specific application of rhBMP-2, this indication may be off-label. A more recently used technique is the addition of accessory supplemental rods, which may reduce primary rod stress and risk of implant failure/pseudarthrosis. ${ }^{35}$ Prior to closure, we decorticate the posterolateral elements for re-arthrodesis, again using off-label rhBMP-2, morselized local bone, and cancellous allograft bone. 
TABLE 1. Demographics, comorbidities, and lumbar spine surgery history

\begin{tabular}{|c|c|c|c|c|c|c|c|c|}
\hline $\begin{array}{l}\text { Case } \\
\text { No. }\end{array}$ & $\begin{array}{l}\text { Age } \\
\text { (yrs), } \\
\text { Sex }\end{array}$ & $\begin{array}{c}\text { BMl } \\
\left(\mathrm{kg} / \mathrm{m}^{2}\right)\end{array}$ & $\begin{array}{l}\text { PI } \\
\left({ }^{\circ}\right)\end{array}$ & Comorbidities & Osteoporosis \& Tx & $\begin{array}{l}\text { Prior Lumbar } \\
\text { Surgery }\end{array}$ & $\begin{array}{l}\text { Index Lumbar } \\
\text { Pathology }\end{array}$ & Index LS Arthrodesis \\
\hline 1 & $80, \mathrm{M}$ & 25.3 & 62 & $\begin{array}{l}\text { Amyloidosis, atrial } \\
\text { fibrillation, CHF }\end{array}$ & None & $\begin{array}{l}\text { Multiple decom- } \\
\text { pressions }\end{array}$ & Spinal stenosis & $\begin{array}{l}\text { L2-S1 PSIF, PCO (L2- } \\
\text { S1), TLIF (L5-S1) }\end{array}$ \\
\hline 2 & $79, \mathrm{~F}$ & $\begin{array}{c}31.4 \\
\text { (obese) }\end{array}$ & 69 & $\begin{array}{l}\text { Cardiomyopathy, } \\
\text { hypothyroidism }\end{array}$ & $\begin{array}{c}\text { Hx of osteoporosis, DEXA hip } \\
\text { T-score }-2.4 \text {, no recent Tx }\end{array}$ & $\begin{array}{l}\text { Decompression } \\
\quad \& \text { fusion }\end{array}$ & $\begin{array}{l}\text { Failed back syn- } \\
\text { drome, PJK }\end{array}$ & T10-S1 PSIF \\
\hline 3 & $64, F$ & $\begin{array}{c}30.2 \\
\text { (obese) }\end{array}$ & 62 & Hypothyroidism & None & Multiple fusions & $\begin{array}{l}\text { Degenerative } \\
\text { scoliosis }\end{array}$ & $\begin{array}{l}\text { T3-S1 PSIF, PCO } \\
\text { (T11-L4) }\end{array}$ \\
\hline 4 & $79, \mathrm{~F}$ & 26.2 & 75 & RA, hypothyroidism & $\begin{array}{l}\text { Hx of osteoporosis, no recent } \\
\text { DEXA or Tx }\end{array}$ & Decom & Spinal stenosis & L2-S1 PSIF, PCO (L3-5) \\
\hline 5 & $70, \mathrm{M}$ & $\begin{array}{c}43.7 \\
\text { (obese) }\end{array}$ & 69 & $\begin{array}{l}\text { Coronary artery } \\
\text { disease, CABG }\end{array}$ & None & Multiple fusions & $\begin{array}{l}\text { Spinal stenosis, } \\
\text { PJK }\end{array}$ & $\begin{array}{l}\text { T10-S1 PSIF, PCO } \\
\text { (T12-L2) }\end{array}$ \\
\hline 6 & $70, \mathrm{M}$ & $\begin{array}{c}30.7 \\
\text { (obese) }\end{array}$ & 63 & Pemphigus & $\begin{array}{l}\text { Osteopenia, DEXA hip T-score } \\
\text {-1.3, no recent Tx }\end{array}$ & Decompression & Spinal stenosis & $\begin{array}{l}\text { L4-S1 PSIF, TLIF (L4-5, } \\
\text { L5-S1) }\end{array}$ \\
\hline 7 & $66, \mathrm{M}$ & $\begin{array}{c}34.3 \\
\text { (obese) }\end{array}$ & 75 & $\begin{array}{l}\text { COPD, current } \\
\text { smoker }\end{array}$ & $\begin{array}{c}\text { Hx of osteoporosis, DEXA hip } \\
\text { T-score }-1.0 \text {, teriparatide }\end{array}$ & None $^{*}$ & Spinal stenosis & $\begin{array}{l}\text { L5-S1 PSIF, TLIF (L5- } \\
\text { S1) }\end{array}$ \\
\hline 8 & $76, \mathrm{~F}$ & 21.9 & 71 & Hypothyroidism & $\begin{array}{l}\text { Osteoporosis, no recent DEXA, } \\
\text { calcitonin nasal spray }\end{array}$ & None ${ }^{*}$ & $\begin{array}{l}\text { High-grade spon- } \\
\text { dylolisthesis }\end{array}$ & $\begin{array}{l}\text { L4-S1 PSIF, L5 Gill lami- } \\
\text { nectomy, TLIF (L5-S1) }\end{array}$ \\
\hline 9 & $69, \mathrm{~F}$ & 26.1 & 71 & $\begin{array}{l}\text { Hyperparathyroidism, } \\
\text { hypothyroidism, RA } \\
\text { (on prednisone) }\end{array}$ & $\begin{array}{l}\text { Steroid-induced osteoporosis, } \\
\text { DEXA hip T-score }-1.6 \text {, wrist } \\
\text { T-score }-1.5 \text {, alendronate }\end{array}$ & None $^{*}$ & Spinal stenosis & $\begin{array}{l}\text { L4-S1 PSIF, TLIF (L4-5, } \\
\text { L5-S1) }\end{array}$ \\
\hline
\end{tabular}

$\mathrm{CABG}=$ coronary artery bypass graft; $\mathrm{CHF}=$ congestive heart failure; $\mathrm{COPD}=$ chronic obstructive pulmonary disease; $\mathrm{Hx}=$ history; $\mathrm{PCO}=$ posterior column osteotomy; PSIF = posterior spinal instrumentation and fusion; RA = rheumatoid arthritis; Tx = treatment.

${ }^{*}$ No history of lumbar spine surgery prior to index LS arthrodesis could be determined from medical charts and imaging.

\section{Electronic Literature Review and Statistical Analysis}

A descriptive analysis of the clinical and radiographic data was performed to identify any trends, commonalities, and/or associations that may inform management of this uncommon complication. We then reviewed the literature to identify articles detailing cases of sacral insufficiency fractures after posterior instrumented LS arthrodesis. Standalone anterior cases (e.g., anterior interbody arthrodesis of L5-S1) were excluded. Articles describing pelvic fractures without a sacral component were also excluded. The literature review was performed by querying PubMed using the search terms "sacral," "fracture," "lumbosacral," and "arthrodesis" for relevant articles up to the year 2019. The references of systematic reviews were also reviewed for additional sources. Articles detailing original cases were then classified according to the authors' treatment/ management (i.e., operative vs nonoperative) and tabulated for descriptive analysis.

\section{Results}

\section{Patient Characteristics and Index LS Arthrodesis}

Nine patients had a minimum 6-month follow-up and were included in the study. Of note, 4 patients without prior LS arthrodesis were excluded for alternate etiology of sacral fracture (i.e., trauma or infection). Demographics, comorbidities, and lumbar spine surgery history are listed in Table 1 for each patient who met the inclusion criteria. The mean patient age at the time of salvage lumbopelvic fixation surgery was $73 \pm 6$ years. Five patients (56\%) were women, and the mean BMI was $30 \pm 6 \mathrm{~kg} / \mathrm{m}^{2}$. Assessment of standing long-cassette radiographic films demonstrated a mean PI of $69^{\circ} \pm 5^{\circ}$. Also, the baseline mean PI-LL mismatch magnitude measured $13^{\circ}$ for patients with available preoperative imaging. The most common comorbidities included osteopenia/osteoporosis (6 patients; mean DEXA hip T-score $-1.6 \pm 0.5$ ), obesity (5 patients), and hypothyroidism (5 patients). Index LS arthrodesis operations were performed for spinal stenosis (6 patients), proximal junctional kyphosis (PJK)/failure (2 patients), degenerative scoliosis (1 patient), and high-grade spondylolisthesis (1 patient). The distal instrumented vertebral level was $\mathrm{S} 1$ for all patients. Six patients had a lumbar upper-most instrumented vertebra (UIV). Three patients had long-segment fusions ( $\geq 6$ levels) with thoracic UIV. L5-S1 interbody arthrodesis/transforaminal lumbar interbody fusion (TLIF).

\section{Sacral Fracture Symptoms and Radiographic Findings}

Symptoms of sacral insufficiency fracture, onset/timing, radiographic findings, and salvage lumbopelvic fixation surgeries are listed in Table 2. All patients presented with persistent back/leg pain and 3 patients had neurological deficits (dorsiflexion $[\mathrm{DF}] /$ plantarflexion $[\mathrm{PF}]$ weakness). For 7 patients, symptom onset was within 4 weeks (mean 3 weeks) of index LS arthrodesis; this prompted CT imaging at a mean postoperative interval of 6 weeks. The remaining 2 patients ( 3 and 5) had delayed presentation with symptom onset at 5.5 and 1 years postoperatively, respectively. All sacral fractures were closely adjacent to or involved S1 screws and traversed the spinal canal (Denis zone III). There was H-type, U-type, or T-type fracture 


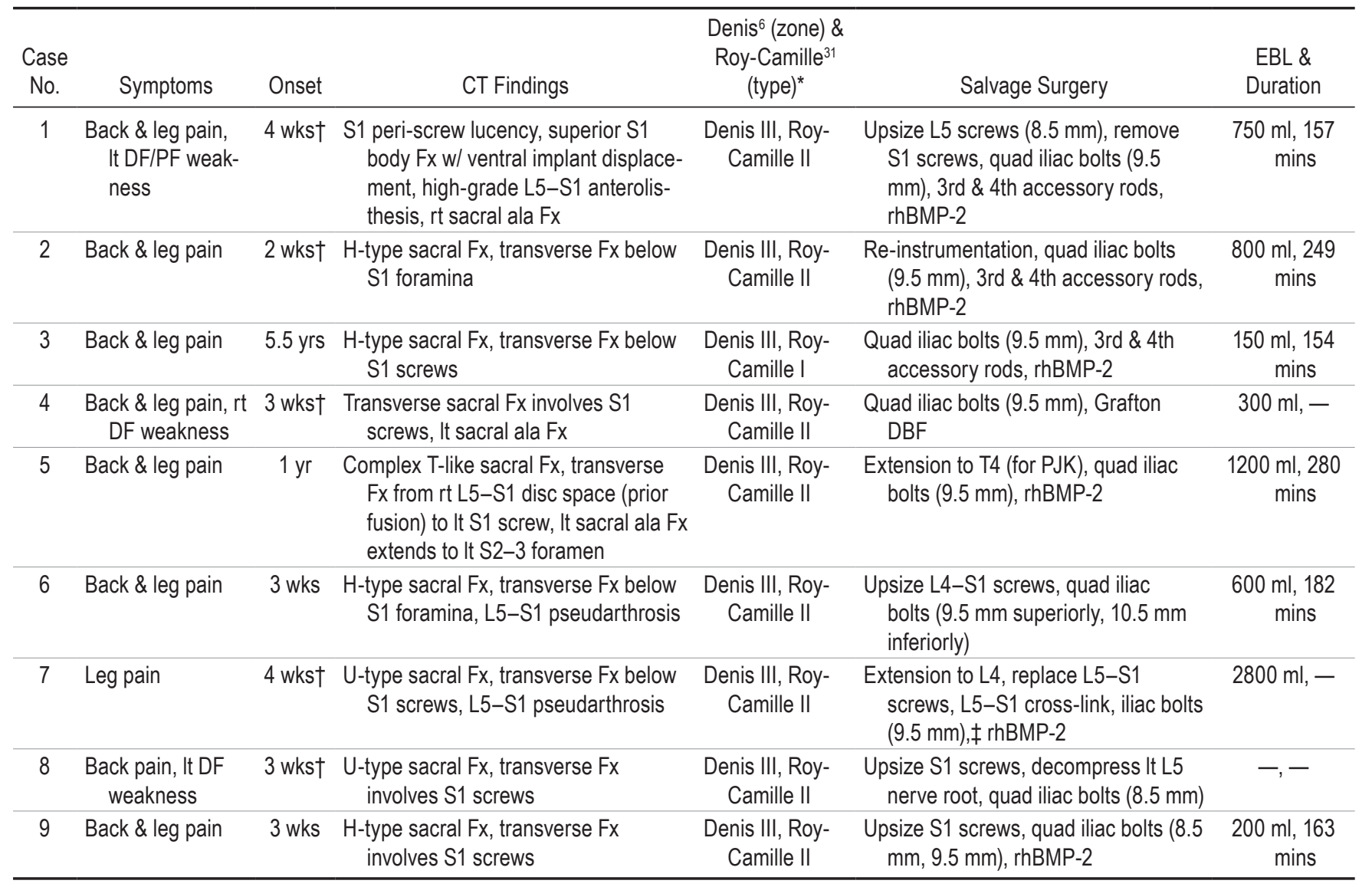

$\mathrm{DBF}=$ demineralized bone fiber; $\mathrm{EBL}=$ estimated blood loss; $\mathrm{Fx}=$ fracture.

${ }^{*}$ Roy-Camille et al. described type II injuries with posterior displacement from flexion loading; however, for this study we categorized angulated sacral fractures with anterior translation (without complete slip) as Roy-Camille type II. ${ }^{31}$

$\dagger$ Patients endorsed history of recent ground-level fall.

$\ddagger$ Bilateral single iliac screws rather than quad iliac bolts.

shape morphology in 7 patients. Fractures were Roy-Camille type II (anterior displacement with kyphosis; 8 patients) and Roy-Camille type I (simple focal kyphosis; 1 patient). Mean preoperative sacral fracture anterolisthesis and kyphotic angulation were $8 \pm 6 \mathrm{~mm}$ and $11^{\circ} \pm 4^{\circ}$, respectively (Table 3 ). Key images from sagittal and coronal $\mathrm{CT}$ reconstructions demonstrate sacral fracture lines for each patient (Fig. 2).

\section{Salvage Lumbopelvic Fixation Surgery}

All salvage surgeries were posterior-only with distal extension of instrumentation for rigid pelvic fixation using bilateral iliac screws (Table 2). Bilateral dual iliac screws (i.e., quad iliac bolts) were utilized in 8 cases. S1 pedicle screws were removed or replaced (upsized when possible) in 1 patient and 5 patients, respectively. Accessory supplemental rods were utilized in 3 cases to reduce primary rod stress; 6 operations included off-label use of rhBMP-2. Two patients underwent proximal extension of instrumentation: patient 7 had proximal extension to L4 due to extensive LS implant failure and pseudarthrosis, and patient 5 underwent proximal extension to T4 for cor- rection of PJK. The mean operative duration was 3.3 hours and mean estimated blood loss was $850 \mathrm{ml}$.

\section{Clinical Outcomes, Radiographic Correction, and Complications}

Table 3 lists outcomes and complications after salvage lumbopelvic fixation surgery. Preoperative back/leg pain and lower extremity weakness improved after surgery for all patients, and in some cases there was complete resolution (mean clinical follow-up duration 35 months [range 8-96 months]). All sacral fractures were evaluated postoperatively with CT or plain radiographs (Fig. 2), which demonstrated adequate healing at last radiographic follow-up (mean radiographic follow-up duration 29 months [range 8-90 months]). Sacral fracture anterolisthesis and kyphotic angulation improved after surgery and were $4 \pm$ $4 \mathrm{~mm}$ and $5^{\circ} \pm 6^{\circ}$, respectively. Two patients underwent revision surgery for rod fracture (patient 5 at 1 year postoperatively, patient 6 at 2 years postoperatively). No other salvage lumbopelvic fixation surgical complications warranting revision were identified. 
TABLE 3. Outcomes, sacral fracture measurements, and complications

\begin{tabular}{|c|c|c|c|c|c|c|}
\hline $\begin{array}{l}\text { Case } \\
\text { No. }\end{array}$ & Clinical Outcome & $\begin{array}{l}\text { Clinical } \\
\text { FU }\end{array}$ & Sacral Fx* & $\begin{array}{c}\text { Sacral Fx } \\
\text { Anterolisthesis }^{16}\end{array}$ & $\begin{array}{l}\text { Sacral Fx } \\
\text { Angulation }^{16}\end{array}$ & Complications \\
\hline 1 & $\begin{array}{l}\text { Pain \& strength } \\
\text { improved }\end{array}$ & $8 \mathrm{mos}$ & $\begin{array}{l}\text { Healed on CT (8 } \\
\text { mos) }\end{array}$ & $\begin{array}{l}\text { Preop } 17 \mathrm{~mm} \text {, } \\
\text { postop } 0 \mathrm{~mm}\end{array}$ & $\begin{array}{l}\text { Preop } 10^{\circ} \\
\text { postop } 0^{\circ}\end{array}$ & $\begin{array}{l}\text { Aspiration pneumonia, inadequate nutrition } \\
\text { requiring PEG tube }\end{array}$ \\
\hline 2 & Pain improved & $10 \mathrm{mos}$ & $\begin{array}{l}\text { Healed on XR (10 } \\
\text { mos) }\end{array}$ & $\begin{array}{l}\text { Preop } 3 \mathrm{~mm}, \\
\text { postop no CT }\end{array}$ & $\begin{array}{l}\text { Preop } 12^{\circ} \text {, postop } \\
\text { no CT }\end{array}$ & None \\
\hline 3 & Pain resolved & $1 \mathrm{yr}$ & $\begin{array}{l}\text { Healed on XR, } \\
(1 \mathrm{yr})\end{array}$ & $\begin{array}{l}\text { Preop } 0 \mathrm{~mm}, \\
\text { postop no CT }\end{array}$ & $\begin{array}{l}\text { Preop } 15^{\circ} \text {, postop } \\
\text { no CT }\end{array}$ & None \\
\hline 4 & $\begin{array}{l}\text { Pain resolved, } \\
\text { strength improved }\end{array}$ & $1 \mathrm{yr}$ & $\begin{array}{l}\text { Healed on XR, } \\
(1 \mathrm{yr})\end{array}$ & $\begin{array}{l}\text { Preop } 4 \mathrm{~mm}, \\
\text { postop no CT }\end{array}$ & $\begin{array}{l}\text { Preop } 9^{\circ} \text {, postop } \\
\text { no CT }\end{array}$ & None \\
\hline 5 & Pain improved & $2 \mathrm{yrs}$ & $\begin{array}{l}\text { Healed on CT } \\
\quad(1.5 \mathrm{yrs})\end{array}$ & $\begin{array}{l}\text { Preop } 17 \mathrm{~mm}, \\
\text { postop } 3 \mathrm{~mm}\end{array}$ & $\begin{array}{l}\text { Preop } 6^{\circ} \text {, postop } \\
0^{\circ}\end{array}$ & $\begin{array}{l}\text { S1-lliac rod Fx (after MVA) requiring revision } \\
\text { surgery approximately } 1 \text { yr postop }\end{array}$ \\
\hline 6 & Pain improved & $4 \mathrm{yrs}$ & $\begin{array}{l}\text { Healed on CT } \\
\quad(1.5 \mathrm{yrs})\end{array}$ & $\begin{array}{l}\text { Preop } 4 \mathrm{~mm}, \\
\text { postop } 3 \mathrm{~mm}\end{array}$ & $\begin{array}{l}\text { Preop } 18^{\circ} \text {, postop } \\
12^{\circ}\end{array}$ & $\begin{array}{l}\text { S1-lliac rod Fx requiring revision surgery } \\
\text { approximately } 2 \text { yrs postop }\end{array}$ \\
\hline 7 & Pain resolved & $4 \mathrm{yrs}$ & $\begin{array}{l}\text { Early CT,† healed } \\
\text { on XR (4 yrs) }\end{array}$ & $\begin{array}{l}\text { Preop } 9 \mathrm{~mm} \text {, post- } \\
\text { op } 11 \mathrm{~mm} \dagger\end{array}$ & $\begin{array}{l}\text { Preop } 8^{\circ} \text {, postop } \\
11^{\circ} \dagger\end{array}$ & Pneumonia \\
\hline 8 & $\begin{array}{l}\text { Pain \& weakness } \\
\text { resolved }\end{array}$ & $8 \mathrm{yrs}$ & $\begin{array}{l}\text { Healed on CT, } \\
\quad(7.5 \mathrm{yrs})\end{array}$ & $\begin{array}{l}\text { Preop } 5 \mathrm{~mm} \text {, } \\
\text { postop } 0 \mathrm{~mm}\end{array}$ & $\begin{array}{l}\text { Preop } 11^{\circ} \text {, postop } \\
0^{\circ}\end{array}$ & None \\
\hline 9 & Pain resolved & $5 \mathrm{yrs}$ & $\begin{array}{l}\text { Healed on CT } \\
\quad(3.5 \mathrm{yrs})\end{array}$ & $\begin{array}{l}\text { Preop } 10 \mathrm{~mm}, \\
\text { postop } 8 \mathrm{~mm}\end{array}$ & $\begin{array}{l}\text { Preop } 8^{\circ} \text {, postop } \\
8^{\circ}\end{array}$ & None \\
\hline
\end{tabular}

$\mathrm{FU}=$ follow-up; $\mathrm{MVA}$ = motor vehicle accident; $\mathrm{PEG}$ = percutaneous endoscopic gastrostomy; $\mathrm{XR}=$ radiography.

* Postoperative time interval of final follow-up CT (or radiography if no CT) is listed in parentheses.

† Patient 7 had early postoperative CT (approximately 1-month postoperatively when presented with pneumonia) with slightly worse anterior translation and angulation of the sacral fracture, but follow-up radiographs demonstrated fracture healing and the patient's pain completely resolved.

\section{Literature Review}

A total of 17 articles describing 87 cases were included in the literature review. Tables 4 and 5 list the articles subdivided by either operative $(n=10)$ or nonoperative $(n=7)$ management, respectively. All included studies were case reports, case series, or retrospective case-control studies. There were no randomized prospective studies. Four published studies reported incidence rates of this complication. ${ }^{14,22,26,27}$ Meredith et al. reported an overall incidence rate of $6.1 \%$, with a higher rate of $14.5 \%$ for index LS arthrodesis $>3$ levels (compared to $1.2 \%$ for LS arthrodesis $\leq 3$ levels). ${ }^{22}$ Klineberg et al. reported incidence rates of $1.3 \%$ and $3.1 \%$ for short-segment and long-segment instrumentation, respectively. ${ }^{14}$ Papadopoulos et al. reported an incidence rate of $4.4 \%$ for long thoracolumbosacral fusions. ${ }^{27}$ Odate et al. reported an incidence rate of $4.3 \%$ in long LS instrumented fusions from L2 or above. ${ }^{26}$ Two published studies with retrospective comparative designs (Odate et al. and Meredith et al.) identified potential positive risk factors for this complication (i.e., osteoporosis, LS arthrodesis with $>3$ fused levels, high PI, high PI-LL mismatch). ${ }^{22,26}$ These same two retrospective comparative studies recommended prophylactic extension of the fusion construct to the iliac wings in high-risk patients. ${ }^{22,26}$ There was a single published study that compared operative and nonoperative cohorts to identify possible surgical indications (i.e., sacral fracture anterolisthesis $>2 \mathrm{~mm}$ and kyphotic angulation $\left.>5^{\circ}\right) .{ }^{22}$

\section{Discussion}

The results of our literature review suggest that sacral insufficiency fractures are an uncommon postoperative complication following posterior segmental instrumented LS arthrodesis. ${ }^{14,22,26,27}$ Incidence rates for this complication have been reported from $1.2 \%$ to $14.5 \% .^{14,22,26,27}$ The higher incidence rates correspond to studies with long fusion constructs, suggesting construct length as a potential risk factor. ${ }^{22}$ Other potential risk factors for postoperative sacral fractures following LS fusion include advanced age, female sex, obesity, osteopenia/osteoporosis, chronic corticosteroid use, postoperative sagittal imbalance (imbalance may be exacerbated by the fracture), and L5-S1 pseudarthrosis. ${ }^{11,14,15,18,20,22,27,33,37-39}$ Note that many of these risk factors are similar with or without spinal instrumentation/arthrodesis and reflect poor host bone quality. ${ }^{22}$ Also, some prior literature supported obesity as a protective factor against osteoporotic fractures, $, 5,10$ but growing evidence suggests obesity may be a risk factor associated with certain fractures, including postoperative sacral insufficiency fracture. . $022,27,38,39$ In a recently published study, Salzmann et al. demonstrated that obesity was associated with approximately six times higher odds of this complication. ${ }^{32}$ Our study cohort had many of these risk factors, including advanced age (mean $73 \pm 6$ years), obesity (mean BMI 30 $\pm 6 \mathrm{~kg} / \mathrm{m}^{2}$ ), osteopenia/osteoporosis (6 patients), long LS fusions to L2 or above (5 patients), and chronic steroid use (1 patient).

In a retrospective comparative study, Odate et al. investigated spinopelvic parameters for potential correlations with sacral fracture development after LS arthrodesis. ${ }^{26}$ The study demonstrated that high PI and larger PI-LL mismatch (both baseline and postoperative measurements) were positive risk factors for this complication. ${ }^{26}$ In com- 


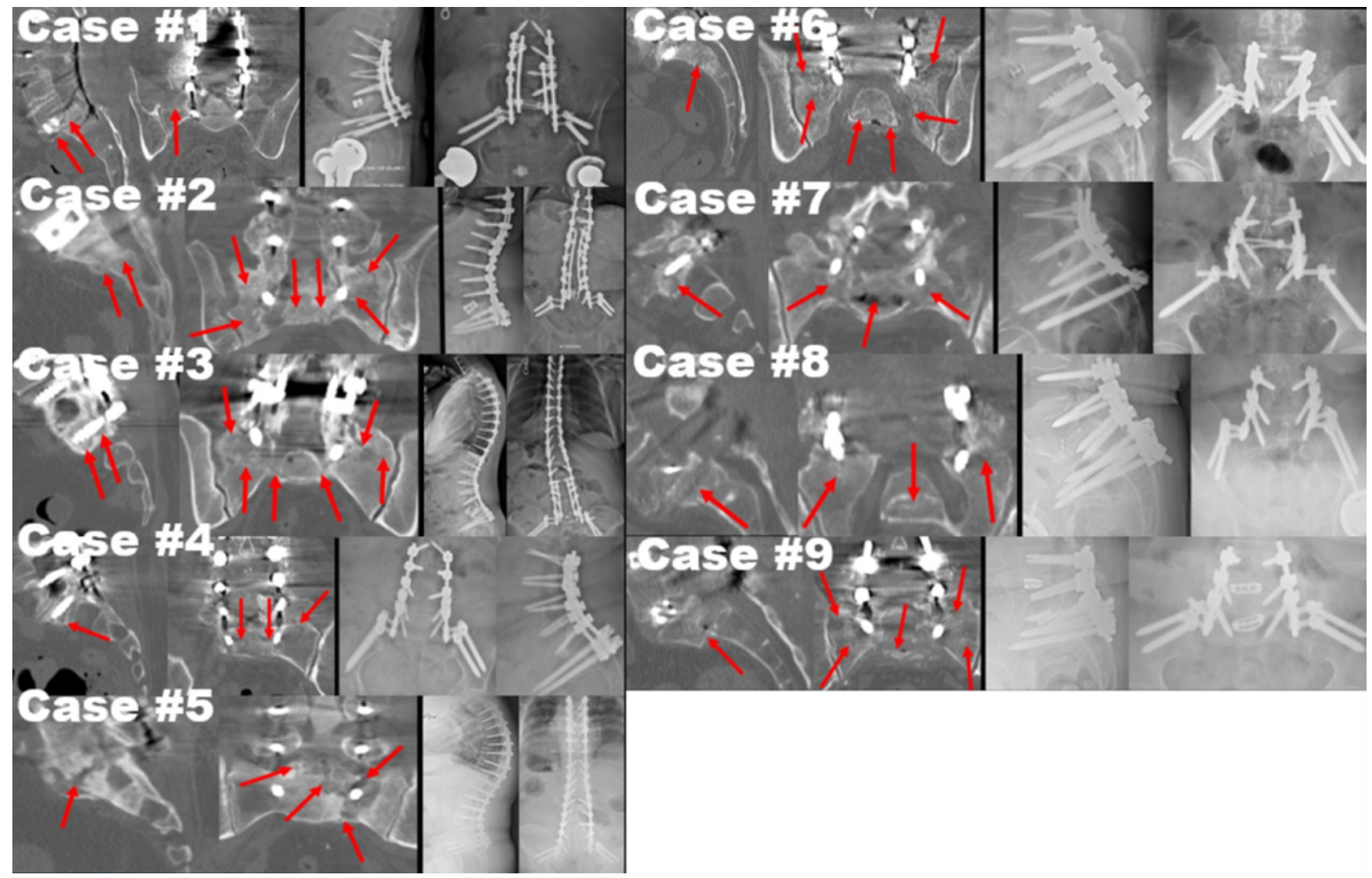

FIG. 2. Sacral fracture CT reconstructions and salvage lumbopelvic fixation postoperative radiographs. This figure demonstrates sacral fracture lines on CT reconstruction images (arrows) and salvage lumbopelvic fixation postoperative radiographs for patients $1-9$, respectively. Further details of CT fracture characteristics, relevant fracture classifications and measurements, and salvage revision surgery are indicated in Tables 2 and 3 . Figure is available in color online only.

parison to reported normal values of PI in the elderly (age $>60$ years, PI $\left.51^{\circ} \pm 9^{\circ}\right),{ }^{34}$ our sacral fracture study patients had higher mean PI $\left(69^{\circ} \pm 5^{\circ}\right)$. Other authors have also reported higher mean PI $\left(64^{\circ}-73^{\circ}\right)$ for patients with this complication. ${ }^{22,26,33,38}$ Accordingly, Odate et al. recommended prophylactic iliosacral fixation to protect the sacrum in patients with high PI preparing for LS arthrodesis. ${ }^{26}$ Recently, Salzmann et al. provided evidence suggesting BMI and sex, more than pelvic parameters and bone mineral density, were risk factors for postoperative sacral fractures. ${ }^{32}$

In our study, presenting symptoms included back/ leg pain (all 9 patients) and lower extremity weakness (3 patients). Symptoms most commonly occurred within 4 weeks of index LS arthrodesis. This prompted CT imaging for definitive fracture diagnoses at an average of approximately 6 weeks postoperatively. Our results are consistent with other published studies (i.e., most sacral fracture patients present with back and/or leg pain within 3 months of index LS arthrodesis surgery)..$^{14,37-39} \mathrm{It}$ is important to note that plain film radiographs often poorly demonstrate these fractures; therefore, a high index of suspicion, even in the setting of negative radiographs, should prompt further investigation with high-resolution $\mathrm{CT}$ with $3 \mathrm{D}$ reconstructions. ${ }^{13,14,22,27,37-39}$ Delays in diagnosis and/or treatment of this complication can have potentially significant conse- quences for elderly spine patients unable to tolerate prolonged immobilization from intractable pain.

CT imaging of our study cohort demonstrated a characteristic fracture pattern with transverse components through the sacral body (Denis zone III), involvement of sacral S1 screws (peri-screw lucency, pseudarthrosis, or adjacent-fracture components), and vertical fracture lines predominantly within the sacral ala. H-, U-, or T-type shape morphology (due to various combinations of transverse/horizontal and vertical fracture lines) were best visualized on coronal CT image reconstructions. ${ }^{39}$ Nearly all fractures (except patient 3) had incomplete anterior translation with kyphotic angulation (i.e., Roy-Camille type II) that was best appreciated on sagittal CT image reconstructions. ${ }^{16,39}$ In contrast to the fracture patterns demonstrated in this study, sacral insufficiency fractures without prior LS arthrodesis typically involve one or both sacral ala with fracture lines that are parallel to the sacroiliac joints. ${ }^{1}$ Wilde et al. also noted distinct radiological features and differences between sacral insufficiency fractures after LS fusion compared to more common osteoporotic sacral stress fractures. ${ }^{39}$

\section{Proposed Management Algorithm}

Based on our study results and literature review, we 
TABLE 4. Operative management of sacral insufficiency fractures after posterior instrumented lumbosacral arthrodesis

\begin{tabular}{|c|c|c|c|c|c|}
\hline Authors \& Year & $\begin{array}{l}\text { Operative } \\
\text { Patients* }\end{array}$ & Salvage Surgery & $\begin{array}{l}\text { Time After LS Fusion to } \\
\text { Presentation/Diagnosis }\end{array}$ & Surgical Indications & Surgical Outcomes \\
\hline $\begin{array}{l}\text { Noh \& Chedid, } \\
\quad 2016\end{array}$ & 1 op & Direct S1-2 screws across Fx & $6 \mathrm{mos}$ & $\begin{array}{l}\text { Refractory pain, S1-2 Fx } \\
\text { nonunion }\end{array}$ & Fx healing, symptom relief \\
\hline $\begin{array}{l}\text { Scemama et } \\
\text { al., } 2016\end{array}$ & 2 opt & $\begin{array}{l}\text { lliac screw fixation } \pm \text { anterior } \\
\text { interbody cage }\end{array}$ & 2 wks, 2 mos & Refractory pain, L5 deficit & $\begin{array}{r}\text { Healed Fx, pain relief, } \\
\text { persistent } L 5 \text { deficit }\end{array}$ \\
\hline $\begin{array}{l}\text { Wang et al., } \\
2016\end{array}$ & 1 op & Iliac screw fixation & $1 \mathrm{wk}$ & $\begin{array}{l}\text { Refractory pain, lower extremity } \\
\text { weakness }\end{array}$ & $\begin{array}{l}\text { Pain relief, strength } \\
\text { improvement }\end{array}$ \\
\hline $\begin{array}{l}\text { Meredith et al., } \\
2013\end{array}$ & $\begin{array}{l}16 \text { op (8 } \\
\text { nonop) }\end{array}$ & $\begin{array}{l}\text { lliac screw fixation } \pm \text { S2 screws } \\
\pm \text { anterior interbody cage }\end{array}$ & $\begin{array}{l}\text { Mean } 4.3 \text { mos } \\
(63 \% \text { w/in } 3 \text { mos })\end{array}$ & $\begin{array}{l}\text { Refractory pain, neurological } \\
\text { deficit, Fx nonunion }\end{array}$ & $\begin{array}{l}2 \text { patients w/ persistent } \\
\text { deficits }\end{array}$ \\
\hline $\begin{array}{l}\text { Wilde et al., } \\
2011\end{array}$ & $\begin{array}{r}12 \text { op (11 } \\
\text { nonop) }\end{array}$ & $\begin{array}{l}\text { Transiliac fixation, transsacral } \\
\text { cage }\end{array}$ & Most w/in 3 mos & Refractory pain, Fx nonunion & - \\
\hline $\begin{array}{l}\text { Klineberg et } \\
\text { al., } 2008\end{array}$ & $\begin{array}{l}6 \text { op (3 } \\
\text { nonop) }\end{array}$ & Iliac screw fixation & Mean 5 wks & $\begin{array}{l}\text { Refractory pain, neurologic } \\
\text { deficit, severe displacement, } \\
\text { Fx nonunion }\end{array}$ & Healed Fx, symptom relief \\
\hline $\begin{array}{l}\text { Papadopoulos } \\
\text { et al., } 2008\end{array}$ & $\begin{array}{l}5 \text { op (long- } \\
\text { segment } \\
\text { fusions) }\end{array}$ & $\begin{array}{l}\text { lliac screw fixation } \pm \text { S2 screws } \\
\pm \text { anterior debridement or } \\
\text { cage }\end{array}$ & Mean 4.6 mos & $\begin{array}{l}\text { Refractory pain, sagittal de- } \\
\text { compensation, Fx nonunion, } \\
\text { L5-S1 pseudarthrosis }\end{array}$ & Fx healing, symptom relief \\
\hline $\begin{array}{l}\text { Vavken \& Kre- } \\
\text { pler, } 2008\end{array}$ & $\begin{array}{l}1 \text { op (3 } \\
\text { nonop) }\end{array}$ & $\begin{array}{l}\text { Extension to S2, iliac screw } \\
\text { fixation }\end{array}$ & 2 wks & Refractory pain, Fx nonunion & Healed Fx, symptom relief \\
\hline $\begin{array}{l}\text { Khanna et al., } \\
2004\end{array}$ & $\begin{array}{l}2 \text { op (1 } \\
\text { nonop) }\end{array}$ & $\begin{array}{l}\text { Extension to S2, strut fibular } \\
\text { allograft }\end{array}$ & 6 mos, 6 wks & Refractory pain, Fx nonunion & Healed Fx, symptom relief \\
\hline Bose, 2003 & 1 op & Extension to $\mathrm{S} 4 \mathrm{w} /$ hooks & $3 \mathrm{mos}$ & Refractory pain & Symptom relief \\
\hline
\end{tabular}

* Some series also had conservatively managed patients.

† Third patient had iliac fixation as part of index operation. ${ }^{33}$

‡ Fibular strut allograft spanning the fractured sacrum (at S1) across L5-S1 disk space and into the L5 vertebral body. ${ }^{13}$

propose a management algorithm for treating sacral fractures after LS arthrodesis (Fig. 3). Many studies support an initial trial of nonoperative treatment, which may be appropriate for a subset of patients with less severe fractures and symptoms. ${ }^{8,9,12,20,22,26}$ Nonoperative treatments include bracing/orthotics, activity modification (e.g., initial bedrest followed by progressive mobilization/ambulation), pharmacological pain control, medical treatment for osteoporosis, and gradual physical rehabilitation. ${ }^{8,9,12 \text {, }}$
15,20,26,28 A period of approximately 6 months for nonoperative therapy may be a reasonable time interval to allow fracture healing/union..$^{14,22}$ Interval imaging should be performed during this time period if pain persists or worsens, which could represent increased fracture displacement. If there is minimal symptomatic improvement and persistent pain after a full trial of nonoperative management, then surgical treatment should be considered. The decision to operate is multifactorial and includes assessment of patient

TABLE 5. Nonoperative management of sacral insufficiency fractures after posterior instrumented lumbosacral arthrodesis

\begin{tabular}{|c|c|c|c|c|c|}
\hline Authors \& Year & $\begin{array}{l}\text { No. of } \\
\text { Patients }\end{array}$ & Nonoperative Tx & $\begin{array}{l}\text { Time After LS Fusion to } \\
\text { Presentation/Diagnosis }\end{array}$ & Symptoms & Outcomes \\
\hline Odate et al., 2013 & 5 & $\begin{array}{l}\text { Activity modification, pain man- } \\
\text { agement, hip spica brace }\end{array}$ & 42 days & Buttock pain & $\begin{array}{l}2 \text { patients } w / \text { persistent } \\
\text { buttock pain }\end{array}$ \\
\hline Khan et al., 2005 & 3 & $\begin{array}{l}\text { Rehab, pharmacological pain } \\
\text { management }\end{array}$ & Mean 1 mo & Sacral \& buttock pain & Pain-free \\
\hline Koh et al., 2005 & 1 & $\begin{array}{l}\text { Gradual rehab, brace, pain } \\
\text { management }\end{array}$ & $7 \mathrm{mos}$ & Low-back \& buttock pain & Not reported \\
\hline Pennekamp et al., 2005 & 1 & Activity modification, TLSO & 9 days & Low-back \& buttock pain & Pain-free \\
\hline Elias et al., 2002 & 1 & Brace & 4 mos & Sacral pain, radiculopathy & Pain improved \\
\hline Fourney et al., 2002 & 1 & $\begin{array}{l}\text { TLSO, osteoporosis medical } \\
\text { treatment }\end{array}$ & $1 \mathrm{wk}$ & Low-back \& buttock pain & Pain-free \\
\hline Mathews et al., 2001 & 2 & $\begin{array}{l}\text { Activity modification, gradual } \\
\text { rehab, pain management }\end{array}$ & 2-4 wks & Low-back pain & Pain improved \\
\hline
\end{tabular}

Rehab $=$ rehabilitation; TLSO = thoracolumbosacral orthosis. 

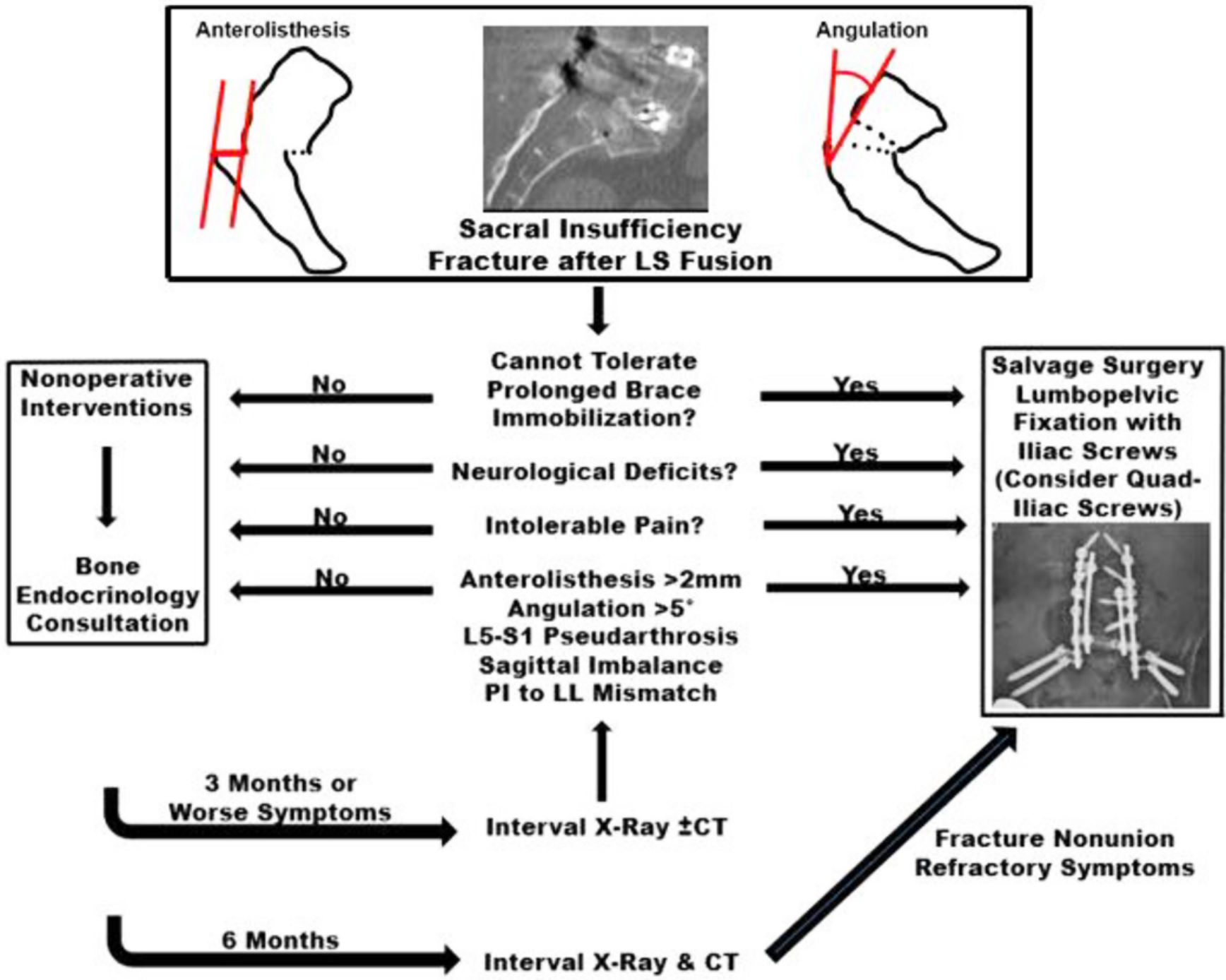

FIG. 3. Management algorithm for sacral insufficiency fracture after posterior instrumented LS arthrodesis. This diagram depicts a proposed treatment algorithm for management of sacral fractures after LS arthrodesis. The decision to pursue surgical treatment is multifactorial. Some potential indications for early surgery include neurological deficits, intolerable pain, patient comorbidities and health conditions that preclude prolonged brace immobilization, and radiographic fracture characteristics such as severe anterior translation or kyphotic angulation. A bone endocrinology consultation and treatment of osteoporosis may be beneficial. During a trial of conservative management, 3-and 6-month follow-up may be prudent to ensure fracture nonunion is not worsening and to assess the patient's clinical response. Ultimately, a focused conversation between surgeon and patient regarding treatment risks, benefits, and alternatives is recommended before pursuing surgery. Figure is available in color online only.

comorbidities and baseline health status. Earlier surgery may be indicated for some patients who cannot tolerate prolonged pain, orthotic devices, brace immobilization, or the prolonged decreased activity often required for a trial of conservative management. ${ }^{22}$ Other patients may present with neurological deficits (patients 1,4, and 8) that could compel surgeons to intervene more expediently. For these patients, careful attention to preoperative CT imaging is important for planning appropriate sacral decompression.

In addition to clinical symptoms, our proposed management algorithm involves radiological assessment, with potential surgical indications that include LS kyphosis with sagittal imbalance or PI-LL mismatch, pseudarthrosis involving the LS junction, and fracture displacement/angu- lation. ${ }^{14,22,27,33,38}$ Initial nonoperative management may be ineffective for some patients because of severe pain and postural decompensation presumably related to fracture motion and sagittal imbalance. ${ }^{14}$ Refractory pain may also be partly attributed to pseudarthrosis involving the LS junction, which can be readily demonstrated on CT imaging (see patients 6 and 7 in our study). ${ }^{27}$ Accordingly, Papadopoulos et al. suggested that the presence of concomitant L5-S1 pseudarthrosis on diagnostic imaging may compel surgeons to intervene earlier. ${ }^{27}$ Finally, assessment of fracture severity using the Kuklo measurement methodology may be another important factor guiding treatment. ${ }^{16}$ Meredith et al. demonstrated that sacral fracture anterolisthesis $>2 \mathrm{~mm}$ and kyphotic angulation $>5^{\circ}$ were significantly 
associated with failure of conservative management. ${ }^{22}$ In a separate study, Klineberg et al. reported surgical candidates with mean preoperative fracture anterolisthesis and kyphosis of $10 \mathrm{~mm}$ and $9.7^{\circ}$, respectively. ${ }^{14}$ In our study, the mean sacral fracture anterolisthesis and kyphotic angulation were $8 \pm 6 \mathrm{~mm}$ and $11^{\circ} \pm 4^{\circ}$, respectively. These radiological parameters can help guide management and should be utilized in the context of clinical symptomatology.

The principle goals of surgical management for this complication include: 1) direct fracture reduction and stabilization for sacral healing/union, 2) decompression of neural impingement, 3) rearthrodesis for potential concomitant LS pseudarthrosis, and 4) correction of LS kyphosis or spinopelvic sagittal malalignment, if pres-

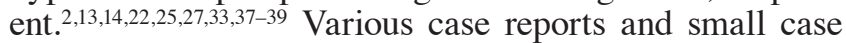
series have demonstrated potentially effective operative techniques to treat this complication. ${ }^{2,13,14,22,25,27,33,37-39}$ For example, Noh and Chedid inserted 50-mm lag screws directly across a transverse sacral fracture (from S2 to S1) and then filled the fracture line with cement. ${ }^{25}$ Khanna et al. performed serial reaming over a guidewire for posterior-to-anterior insertion of strut fibular allograft to span the sacral nonunion, L5-S1 disk space, and L5 vertebral body. ${ }^{13}$ Some surgeons utilized an anterior approach for fracture debridement and/or cage placement when there is extensive bone loss..$^{22,27,33}$ Distal extension of the LS fusion construct with pelvic fixation, via iliac screws or S2-alariliac screws, was also a reported salvage treatment modality. ${ }^{14,22,27,33}$

In this study, we performed lumbopelvic fixation with iliac screws for all patients. Iliac screw fixation can reduce sacral stress and unload force transmission at the LS junction. ${ }^{17,21}$ When possible, we prefer to insert dual iliac screws bilaterally (i.e., quad iliac bolt constructs) to further offload the injured sacrum for optimal healing and bone union. , $7,17,41$ Our results demonstrated symptomatic improvement and fracture healing in all patients: mean sacral fracture anterolisthesis and kyphotic angulation improved from $8 \mathrm{~mm}$ to $4 \mathrm{~mm}$ and $11^{\circ}$ to $5^{\circ}$, respectively. There were no complications directly related to the additional bilateral iliac screw fixation (i.e., third and fourth iliac screws). There were two cases of rod fracture at the S1-iliac connection that required revision; however, one of these rod fractures was likely related to a motor vehicle accident. Overall, we report low complications with our technique of bilateral dual iliac screw fixation as a salvage treatment modality for post-LS fusion sacral fractures.

Bourghli et al. recently reviewed the literature on dual iliac screw constructs and identified three general indications for this technique of spinopelvic fixation: 1) adult deformity correction in osteoporotic patients requiring long fusion constructs or 3-column sacral osteotomies, 2) reduction-stabilization for traumatic U-shape sacral fractures, and 3) stabilization after total sacrectomy in oncology patients. ${ }^{3}$ Our study results provide evidence to suggest sacral insufficiency fracture after LS fusion may be another reasonable indication for dual iliac screw constructs in a subset of appropriately selected patients. However, it is important to be aware of the limitations of this study. First, we performed a retrospective, single-center descriptive analysis with inherent bias from treatment and practice patterns of the senior authors. Our institution provides tertiary-level care and a subset of study patients were referrals from other surgeons. Therefore, the available data set utilized for this study precluded definitive calculation of our institutional rate of sacral fractures after LS arthrodesis. Also, incomplete baseline radiographic data for referred patients may limit assessment of prefracture spinopelvic parameters because sacral fractures have the potential to change PI. ${ }^{22}$ Next, this study lacked available patient-reported outcome scores or a control group for comparison to nonoperative interventions. Also, as with many studies of uncommon complications, our study was limited by small cohort size. To address some of these issues, we reviewed the pertinent literature and supplemented our results with data from other surgeons. However, the literature review produced articles with inconsistent methodology for reporting and assessing outcomes. For example, we identified a single article that reported both pre- and postoperative anterolisthesis and angulation according to Kuklo sacral fracture measurements. ${ }^{14,16}$ Also, many studies did not include spinopelvic parameters such as PI, which is a potential positive risk factor associated with this complication. ${ }^{26}$ We acknowledge that a large, multicenter prospective study would be helpful in addressing these aforementioned limitations. Further revisions to our management algorithm may be needed if future studies identify other variables that potentially impact treatment outcomes for this rare complication.

\section{Conclusions}

Sacral insufficiency fractures are an uncommon postoperative complication that can occur after posterior instrumented LS arthrodesis. Affected patients may complain of persistent back/leg pain and sacral tenderness; frank weakness or neurological deficits are less common. This complication usually occurs within a few months after surgery and can lead to significant morbidity and delayed postoperative recovery in elderly spine patients. Because plain radiographs are often unrevealing, a high index of suspicion and CT imaging are often needed to make the diagnosis. An initial trial of conservative management is reasonable for select patients with less severe fractures. Potential indications for early surgical treatment include intolerable pain, neurological deficits, fracture nonunion with anterolisthesis or kyphotic angulation, concurrent L5-S1 pseudarthrosis, and spinopelvic sagittal malalignment. Our study results provide evidence to suggest lumbopelvic fixation with bilateral dual iliac screws can effectively salvage the index LS fusion, allow sacral fracture healing, and improve clinical symptoms. Patients with positive risk factors for this postoperative complication may benefit from prophylactic lumbopelvic fixation at the time of index LS arthrodesis.

\section{References}

1. Blake SP, Connors AM: Sacral insufficiency fracture. Br J Radiol 77:891-896, 2004

2. Bose B: Fracture of S1-2 after L4-S1 decompression and fusion. Case report and review of the literature. J Neurosurg 99 (3 Suppl):310-312, 2003

3. Bourghli A, Boissiere L, Obeid I: Dual iliac screws in spino- 
pelvic fixation: a systematic review. Eur Spine J 28:20532059, 2019

4. Cooper KL, Beabout JW, Swee RG: Insufficiency fractures of the sacrum. Radiology 156:15-20, 1985

5. De Laet C, Kanis JA, Odén A, Johanson H, Johnell O, Delmas $\mathrm{P}$, et al: Body mass index as a predictor of fracture risk: a meta-analysis. Osteoporos Int 16:1330-1338, 2005

6. Denis F, Davis S, Comfort T: Sacral fractures: an important problem. Retrospective analysis of 236 cases. Clin Orthop Relat Res 227:67-81, 1988

7. Ebata S, Ohba T, Oba H, Haro H: Bilateral dual iliac screws in spinal deformity correction surgery. J Orthop Surg Res 13:260, 2018

8. Elias WJ, Shaffrey ME, Whitehill R: Sacral stress fracture following lumbosacral arthrodesis. Case illustration. J Neurosurg 96 (1 Suppl): 135, 2002

9. Fourney DR, Prabhu SS, Cohen ZR, Gokaslan ZL, Rhines LD: Early sacral stress fracture after reduction of spondylolisthesis and lumbosacral fixation: case report. Neurosurgery 51:1507-1511, 2002

10. Gonnelli S, Caffarelli C, Nuti R: Obesity and fracture risk. Clin Cases Miner Bone Metab 11:9-14, 2014

11. Gotis-Graham I, McGuigan L, Diamond T, Portek I, Quinn $\mathrm{R}$, Sturgess A, et al: Sacral insufficiency fractures in the elderly. J Bone Joint Surg Br 76:882-886, 1994

12. Khan MH, Smith PN, Kang JD: Sacral insufficiency fractures following multilevel instrumented spinal fusion: case report. Spine (Phila Pa 1976) 30:E484-E488, 2005

13. Khanna AJ, Kebaish KM, Ozdemir HM, Cohen DB, Gonzales RA, Kostuik JP: Sacral insufficiency fracture surgically treated by fibular allograft. J Spinal Disord Tech 17:167173,2004

14. Klineberg E, McHenry T, Bellabarba C, Wagner T, Chapman J: Sacral insufficiency fractures caudal to instrumented posterior lumbosacral arthrodesis. Spine (Phila Pa 1976) 33:1806-1811, 2008

15. Koh YD, Kim JO, Lee JJ: Stress fracture of the pelvic wingsacrum after long-level lumbosacral fusion: a case report. Spine (Phila Pa 1976) 30:E161-E163, 2005

16. Kuklo TR, Potter BK, Ludwig SC, Anderson PA, Lindsey RW, Vaccaro AR: Radiographic measurement techniques for sacral fractures consensus statement of the Spine Trauma Study Group. Spine (Phila Pa 1976) 31:1047-1055, 2006

17. Lebwohl NH, Cunningham BW, Dmitriev A, Shimamoto $\mathrm{N}$, Gooch L, Devlin V, et al: Biomechanical comparison of lumbosacral fixation techniques in a calf spine model. Spine (Phila Pa 1976) 27:2312-2320, 2002

18. Lourie H: Spontaneous osteoporotic fracture of the sacrum. An unrecognized syndrome of the elderly. JAMA 248:715717,1982

19. Maeda T, Higashino K, Manabe H, Yamashita K, Hayashi F, Goda Y, et al: Pullout strength of pedicle screws following redirection after lateral or medial wall breach. Spine (Phila Pa 1976) 43:E983-E989, 2018

20. Mathews V, McCance SE, O'Leary PF: Early fracture of the sacrum or pelvis: an unusual complication after multilevel instrumented lumbosacral fusion. Spine (Phila Pa 1976) 26:E571-E575, 2001

21. McCord DH, Cunningham BW, Shono Y, Myers JJ, McAfee PC: Biomechanical analysis of lumbosacral fixation. Spine (Phila Pa 1976) 17 (8 Suppl):S235-S243, 1992

22. Meredith DS, Taher F, Cammisa FP Jr, Girardi FP: Incidence, diagnosis, and management of sacral fractures following multilevel spinal arthrodesis. Spine J 13:1464-1469, 2013

23. Newhouse KE, el-Khoury GY, Buckwalter JA: Occult sacral fractures in osteopenic patients. J Bone Joint Surg Am 74:1472-1477, 1992

24. Nguyen JH, Buell TJ, Wang TR, Mullin JP, Mazur MD,
Garces J, et al: Low rates of complications after spinopelvic fixation with iliac screws in 260 adult patients with a minimum 2-year follow-up. J Neurosurg Spine 30:635-643, 2019

25. Noh T, Chedid MK: A novel technique to repair a transverse sacral fracture in a previously fused lumbosacral spondylolisthesis. Surg Neurol Int 7 (Suppl 38):S914-S916, 2016

26. Odate S, Shikata J, Kimura H, Soeda T: Sacral fracture after instrumented lumbosacral fusion: analysis of risk factors from spinopelvic parameters. Spine (Phila Pa 1976) 38:E223-E229, 2013

27. Papadopoulos EC, Cammisa FP Jr, Girardi FP: Sacral fractures complicating thoracolumbar fusion to the sacrum. Spine (Phila Pa 1976) 33:E699-E707, 2008

28. Pennekamp PH, Kraft CN, Stütz A, Diedrich O: [Sacral fracture as a rare early complication of lumbosacral spondylodesis.] Z Orthop Ihre Grenzgeb 143:591-593, 2005 (German)

29. Polly DW Jr, Orchowski JR, Ellenbogen RG: Revision pedicle screws. Bigger, longer shims - what is best? Spine (Phila Pa 1976) 23:1374-1379, 1998

30. Rawlings CE III, Wilkins RH, Martinez S, Wilkinson RH Jr: Osteoporotic sacral fractures: a clinical study. Neurosurgery 22:72-76, 1988

31. Roy-Camille R, Saillant G, Gagna G, Mazel C: Transverse fracture of the upper sacrum. Suicidal jumper's fracture. Spine (Phila Pa 1976) 10:838-845, 1985

32. Salzmann SN, Ortiz Miller C, Carrino JA, Yang J, Shue J, Sama AA, et al: BMI and gender increase risk of sacral fractures after multilevel instrumented spinal fusion compared with bone mineral density and pelvic parameters. Spine J 19:238-245, 2019

33. Scemama C, D'astorg H, Guigui P: Sacral stress fracture after lumbar and lumbosacral fusion. How to manage it? A proposition based on three cases and literature review. Orthop Traumatol Surg Res 102:261-268, 2016

34. Schwab F, Lafage V, Boyce R, Skalli W, Farcy JP: Gravity line analysis in adult volunteers: age-related correlation with spinal parameters, pelvic parameters, and foot position. Spine (Phila Pa 1976) 31:E959-E967, 2006

35. Smith JS, Shaffrey CI, Bess S, Shamji MF, Brodke D, Lenke LG, et al: Recent and emerging advances in spinal deformity. Neurosurgery 80 (3S):S70-S85, 2017

36. Strange-Vognsen $\mathrm{HH}$, Lebech A: An unusual type of fracture in the upper sacrum. J Orthop Trauma 5:200-203, 1991

37. Vavken P, Krepler P: Sacral fractures after multi-segmental lumbosacral fusion: a series of four cases and systematic review of literature. Eur Spine J 17 (Suppl 2):S285-S290, 2008

38. Wang Y, Liu XY, Li CD, Yi XD, Yu ZR: Surgical treatment of sacral fractures following lumbosacral arthrodesis: case report and literature review. World J Orthop 7:69-73, 2016

39. Wilde GE, Miller TT, Schneider R, Girardi FP: Sacral fractures after lumbosacral fusion: a characteristic fracture pattern. AJR Am J Roentgenol 197:184-188, 2011

40. Wood KB, Schendel MJ, Ogilvie JW, Braun J, Major MC, Malcom JR: Effect of sacral and iliac instrumentation on strains in the pelvis. A biomechanical study. Spine (Phila Pa 1976) 21:1185-1191, 1996

41. Yu BS, Zhuang XM, Zheng ZM, Li ZM, Wang TP, Lu WW: Biomechanical advantages of dual over single iliac screws in lumbo-iliac fixation construct. Eur Spine J 19:1121-1128, 2010

\section{Disclosures}

Dr. Buell reports receiving honoraria from Wolters Kluwer. Dr. Buchholz reports being a consultant for Medtronic, NuVasive, and Siemens. Dr. Yen reports being a consultant to NuVasive. Dr. Shaffrey reports being a consultant to Medtronic, NuVasive, 
Buell et al.

Zimmer-Biomet, and K2M; receiving royalties from Medtronic, NuVasive, and Zimmer-Biomet; and being a stock holder in NuVasive. Dr. Smith reports receiving royalties from Zimmer Biomet and NuVasive; being a consultant for Zimmer Biomet, Cerapedics, NuVasive, K2M, and AlloSource; receiving honoraria from Zimmer Biomet, NuVasive, and K2M; receiving research support from DePuy Synthes, AOSpine, and ISSGF; receiving fellowship support from the NREF and AOSpine; being a stockholder for Alphatec; and being on the editorial boards of the Journal of Neurosurgery: Spine, Neurosurgery, and Operative Neurosurgery.

\section{Author Contributions}

Conception and design: Buell, CI Shaffrey, Smith. Acquisition of data: Buell. Analysis and interpretation of data: all authors. Draft- ing the article: Buell. Critically revising the article: all authors. Reviewed submitted version of manuscript: all authors. Approved the final version of the manuscript on behalf of all authors: Buell. Statistical analysis: Buell. Study supervision: CI Shaffrey, Smith.

\section{Correspondence}

Thomas J. Buell: University of Virginia Health System, Charlottesville, VA. tjb4p@hscmail.mcc.virginia.edu. 